PREPARED FOR THE U.S. DEPARTMENT OF ENERGY, UNDER CONTRACT DE-AC02-76-CHO-3073

PPPL-2824
PPPL-2824

UC-420,427
ANOMALOUS ENERGY EXCHANGE IN THE gBL * AND QUASILINEAR THEORIES
BY

\author{
H.E. MYNICK
}

February 1992

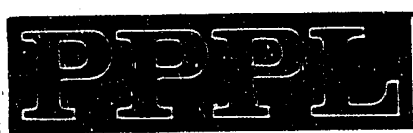

panNCETON

plasma pHYsics

LABofatopr

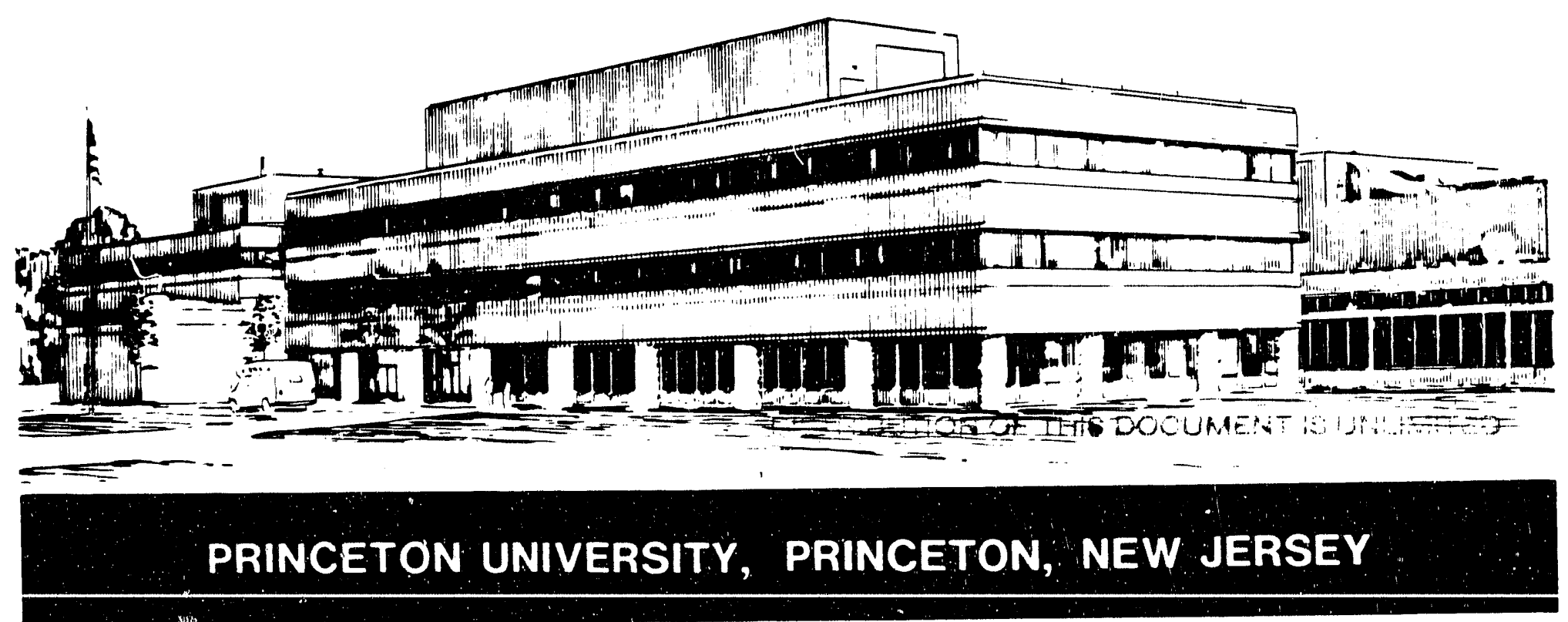




\section{NOTICE}

This report was prepared as an account of work sponsored by an agency of the United States Government. Neither the United States Government nor any agency thereof, nor any of their employees, makes any warranty, express or implied, or assumes any legal liability or responsibility for the accuracy, completeness, or usefulness of any information, apparatus, product, or process disclosed, or represents that its use would not infringe privately owned rights. Reference herein to any specific cornmercial produce, process, or service by trade name, trademark, manufacturer, or otherwise, does not necessarily constitute or imply its endorsement, recommendation, or favoring by the United States Government or any agency thereof. The views and opinions of authors expressed herein do not necessarily state or reflect those of the United States Government or any agency thereof.

\section{NOTICE}

This report has been reproduced directly from the best available copy.

Available to DOE and DOE contractors from the:

Office of Scientific and Technical Information

P.O. Box 62

Oak Ridge, TN 37831;

Prices available from (615) 576-8401.

Available to the public from the:

National Technical Information Service

U.S. Department of Commerce

5285 Port Royal Road

Springfield, Virginia 22161

$703-487-4650$ 


\title{
Anomalous Energy Exchange in the gBL and Quasilinear Theories
}

\author{
H.E.Mynick \\ Plasma Physics Laboratory, Princeton University \\ P.O. Box 451 \\ Princeton, New Jersey 08543-0451, U.S.A.
}

\begin{abstract}
The rate of turbulence-induced energy exchange $\dot{W}_{0}$ between species is computed in the framework of the quasilinear and $\mathrm{gBL}$ transport theories. and the relationship between these two similar theories is thereby elucidated. For both theories, general formal expressions for $\dot{W}_{0}$ are developed, and then applied to the trapped electron mode for illustration. The general expressions for $\dot{W}_{0}$ in the two theories are formally closely related, but can yield predictions of very different magnitude in concrete applications. The fact that quasilinear theory is not valid for saturated steady-state turbulence gives rise to certain peculiarities in its predictions for this normal experimental situation, such as permitting energy to flow from the rooler to the hotter species, even in the limit of thermal equilibrium, where real-space gradients vanish. The gBL theory may be viewed as a modification of quasilinear theory to be valid for steady-state turbulence, keeping extra terms due to the self-consistent back reaction of particles on the fluctuations, which are just such as to eliminate these peculiarities.
\end{abstract}




\section{Introduction}

This paper investigates the relationship between quasilinear ( $\mathrm{ql}$ ) theory ${ }^{1-3}$ and the more recent 'generalized Balescu-Lenard' (gBL) transport theory, ${ }^{6,7}$ two philosophically and formally similar theories with, however, significantly differing physical content. We focus chiefly on the prediction from the two theories of the rate $\dot{W}_{0}$ of turbulent energy exchange between species. This calculation, of potential significance in its own right, ${ }^{8,9}$ also serves as a useful vehicle by which to show new features of the gBL theory. both over ql theory, as well as beyond those applications of the gBL theory already made.

Quasilinear theory forms an important element in the overall structure of plasma theory - the simplest statistical theory describing the selfconsistent interaction of plasma particles and waves, possessing the appropriate set of conservation laws, and an $\mathrm{H}$-theorem (for the particles). As such, the theory consists of two portions, one describing the time evolution of the particle phase-averaged distribution, and the other the evolution of the waves. In its numerous applications to problems of turbulent transport, it is the ql equation for the particle evolution which is focussed upon, providing a simple, explicit prescription for computing the particle transport coefficients for any given spectrum of waves, which are implicitly assumed to evolve according to the ql wave equation.

This implicit assumption presents a difficulty for the relevance of $\mathrm{q}$ l theory to experimental plasmas, however, since it is valid only either during the early, linear growth stage, or for very particular, 'quasilinearly flattened' distributions, for which all the modes have been linearly stabilized. The 
first case does not pertain to the normal experimental situation, however, in which the wave spectrum may usually be characterized as near a quasisteady 'saturated' state, in which nonlinear effects neglected in ql theory are large enough to balance the linear ones. And the second case does not normally pertain either, since these nonlinear effects, rather than ql flattening, probably play a crucial role in establishing the saturation. Schematically, writing the distribution function $f=f_{0}+\delta f$ as the sum of an ensembleaveraged plus a fluctuating part, the averaged and fluctuating parts of the Vlasov equation are

$$
\begin{aligned}
\partial f_{0} / \partial t & =-\partial \cdot\langle\delta \mathbf{E} \delta f\rangle \equiv-\partial \cdot \mathbf{\Gamma} \\
g^{-1} \delta f & =-\delta \mathbf{E} \cdot \partial f_{0}-\delta(\delta \mathbf{E} \cdot \partial \delta f) \equiv g^{-1}\left(\delta f^{(c)}+\delta f^{(i)}\right)
\end{aligned}
$$

where $g$ is the propagator along unperturbed trajectories, and $\partial$ is a momentumspace gradient. For an unmagnetized plasma, $g^{-1} \rightarrow \partial / \partial t+\mathrm{v} \cdot \nabla$, and $\boldsymbol{\partial} \rightarrow e \partial / \partial \mathbf{p}$, with $e$ and $\mathbf{p} \equiv M \mathbf{v}$ the particle charge and momentum, respectively. $\delta f^{(c)}$ is the phase coherent part of $\delta f$, coming from the linear term on the right side of Eq.(2), and $\delta f^{(i)}$ is the 'incoherent noise' portion, ${ }^{4}$ arising from the nonlinear term there. Transport is described by $\Gamma$, the averaged flux in momentum space, and is evaluated by solving Eq.(2) for $\delta f$, and inserting it into the definition of $\Gamma$ in Eq.(1). This yields two parts for the flux, $\Gamma=\Gamma_{\mathbf{D}}+\Gamma_{\mathbf{F}}$. Quasilinear theory neglects the nonlinear term $\delta f^{(i)}$ and so drops the corresponding contribution $\Gamma_{F}$ to $\Gamma$, keeping only the diffusive portion $\Gamma_{\mathbf{D}}=-\mathbf{D} \cdot \partial f_{0}$ arising from $\delta f^{(c)}$. However, for fully-developed turbulence, this nonlinearly-derived term is in general comparable with the coherent term kept, and moreover, its structure is just such as to permit it to assume the role in maintaining conservation laws for steady-state turbu- 
lence performed by the time-varying wave amplitudes in ql theory. ${ }^{4,5}$ Now, instead of energy (for example) being transferred to or from a given species $s_{1}$ (abbreviated to simply 1 hereafter) from each plasma mode $a$ as in ql theory, for a steady-state spectrum, mode a only mediates the exchange of energy from species 1 to some other species 2, but does not change in amplitude, similar to the usual picture for the standard Balescu-Lenard (BL) operator.

The gBL theory ${ }^{6,7}$ may be viewed, on the one hand, as a modification of ql theory to be valid for steady-state spectra, and, on the other, as an analytically manageable theory obtained from fully turbulent forms $s^{4,10}$ for $\Gamma$ by making a simplifying approximation (the 'pseudothermal ansatz ${ }^{\prime 6}$ ) on the exact form for $\Gamma_{F}$, which retains all the appropriate conservation laws and the $\mathrm{H}$-theorem. In this approximation, $\Gamma_{\mathbf{F}}$ has the form $\left(\mathbf{F} f_{0}\right)$, with F the 'dynamic friction,' representing, analogous to the $\mathrm{BL}$ operator, the effects on test particles (or 'macroparticles,' i.e., phase-space granulations) of the self-consistent (turbulent) fluctuations which they themselves drive.

Certain important results of this work can he given and discussed without getting into the formal details of the action-angle formalism, in terms of which the gBL theory is conveniently described, but which is a somewhat nonstandard formal framework. Such results are presented in Sec. II. There. it will be seen that the gBL expression $\dot{W}_{0}^{g B L}$ for $\dot{W}_{0}$ is formally closely related to the ql expression $\dot{W}_{0}^{q l}$, but the retention of $\Gamma_{F}$ yields an additional antisymmetrizing term [cf.Eq.( $\tau)$ ], which can make the predictions for the actual size of $\dot{W}_{0}$ from the two theories very different. To proceed further, in Sec. III we introduce the necessary formalism, and then prove and expand upon the general results stated in Secs. I and II. The focus of this paper 
is interspecies energy exchange, and energy conservation. Formally parallel to the energy conservation theorems in both theories, also introduced in Sec. II, are theorems for conservation of angular momentum $p_{\zeta}$. Because the time rate of change of $p_{\zeta}$ governs the radial particle fluxes $\Gamma$, statements on the relation between the fluxes $\Gamma^{q l}$ and $\Gamma^{g B L}$ analogous to those between $\dot{W}_{0}^{q l}$ and $\dot{W}_{0}^{g B L}$ can be made. For example, as discussed in Sec. IV, the neglect of $\Gamma_{F}$ by ql theory results in a test-particle expression for $\Gamma$, where the collision operator is non-momentum-conserving. As is well known in the special case of neoclassical transport of ions, this flux is much larger than that from the self-consistent calculation, which uses the momentumconserving ( $g B L$ ) operator in which $\Gamma_{F}$ is retained.

Earlier applications ${ }^{6,7}$ of the gBL theory to turbulent transport have made use of fairly crude, generic models for the turbulent spectrum. This has made less clear the comparison between predictions from that theory and ql theory, whose normal application to transport problems considers some specific set of plasma modes of potential significance for turbulent transport. To remedy this deficiency, in Sec. IV, explicit forms are obtained for $\dot{W}_{0}$ for both the $\mathrm{ql}$ and $\mathrm{gBL}$ theories, for the (collisionless) trapped electron mode (TEM). From this 'template' problem, the further application of the gBL theory to other modes of interest should be as direct as for ql theory. Within the context of this application, it will be concretely shown that while formally similar, the $g B L$ prediction for $\dot{W}_{0}$ can be smaller than, comparable to, or larger than the ql prediction, depending upon plasma parameters.

The fact that ql theory is not really applicable to the saturated phase of spectral development results in certain oddities in the ql prediction for $\mid \dot{V}_{0}^{q l}$. One of these is that contrary to what one might expect intuitively, and finds 
for classical (collisional) interspecies exchange, ql theory does not predict that energy "runs downhill," i.e., that it is transferred from the hotter to the cooler species, even for a uniform Maxwellian distribution $f_{0}=f_{M}$, but rather work is done on species 1 in proportion to its contribution to the growth rate (or to the dissipative part $\Delta_{a}^{\prime \prime}(\omega \mid 1)$ of its susceptibility) of the fluctuations [cf.Eq.(10)]. As will be seen in Sec. IV, the additional antisymmetrizing term on the right side of Eq.(7) present in the gBL theory causes that theory to predict turbulently as well as collisionally-exchanged energy to run downhill, in the equilibrium limit $f_{0} \rightarrow f_{M}$.

A related oddity of ql theory, sufficiently independent of formal details that it can be discussed in Sec. II, concerns the nature of its H-theorem. While the ql conservation laws for momentum and energy both involve a sum over contributions from both the particles and the waves, the $\mathrm{ql} \mathrm{H}$ theorem involves only the particle contribution $\dot{S}_{p}$ to the rate of change $\dot{S}$ of entropy for the total system, and, moreover, the ql expression for the wave contribution $\dot{S}_{w}$ in general is of indefinite sign. In contrasi, while the gBL theory also possesses the conservation laws, $\mathrm{H}$-theorem, and Onsager relations ${ }^{5,6}$ there are only particle contributions to any of these.

In Sec. V, we conclude with some discussion summarizing what has been accomplished in the foregoing sections, indicating some of the limitations of the $\mathrm{ql}$ and the $\mathrm{gBL}$ theories, and pointing to possible directions for improvement of the gBL theory. 


\section{Some General Results}

The general relationship between the rates $\dot{W}(1) \equiv\langle\delta \mathrm{E} \cdot \delta \mathrm{j}(1)\rangle$ of turbulent energy exchange in the gBL and ql theories is sufficiently simple [cf.Eq.(7)] that one can write it out at the outset, and prove and clarify it in the later more detailed development. The total energy in species 1 is given by $W(1) \equiv \int d^{6} z_{1} H\left(z_{1}\right) f\left(z_{1}\right)$, with $H(z)=H_{0}(z)+h(z)$ the particle Hamiltonian at phase point $z$, and $f(z)$ the distribution function. $f$ may be solved for in powers of the field amplitude $\phi, f=f_{0}+f_{1}+f_{2}$, plus higher-order terms, which are neglected. $H_{0}$ is the unperturbed, and $h$ is the perturbing, part of $H$. In the gBL theory as well as ql theory, one has $W=W_{0}+W_{2}$, where changes in $W_{0}(1) \equiv \int d^{6} z_{1} H_{0}\left(z_{1}\right) f_{0}\left(z_{1}\right)$ represent irreversible (resonant) work done on species 1 , while $W_{2}$, defined analogously to $W_{0}$, describes the nonresonant particle 'sloshing' energy, and is normally' grouped with the total wave energy $W_{w} \cdot{ }^{3}$ The ql expression for $\dot{W}_{0}$ for species 1 may be writien

$$
\dot{W}_{0}^{q l}(1)=2 \sum_{a} \omega_{a} \frac{\dot{N}_{a} \Delta_{a}^{\prime \prime}\left(\omega_{a} \mid 1\right)}{4 \pi}|\overline{\bar{\phi}}|^{2}(a)
$$

where $|\overline{\bar{\phi}}|^{2}(a)$ is the fluctuation spectrum, labelled by mode index $a$. [For definiteness, one useful model ${ }^{6}$ for the parameters characterizing the plasma modes is $a-\left(\mathbf{k}, r_{a}\right)$, with wavevector $\mathbf{k}$, and mode localization radius $r_{a}$. The precise definition of mode amplitude $\overline{\bar{\phi}}$ will be specified in Sec. IV.] $\Delta_{a}^{\prime \prime}(\omega \mid 1)$ is the contribution from species 1 to the dissipative part $\Delta_{a}^{\prime \prime}(\omega)$ of the dielectric function $\Delta_{a}(\omega)=\Delta_{a}^{\prime}(\omega)+i \Delta_{a}^{\prime \prime}(\omega)=1+\sum_{1} \Delta_{a}(\omega \mid 1)$, evaluated for mode $a$. Similar to Ref. $6, \dot{N}_{a} \equiv \int d \mathbf{x}\left|\dot{\mathrm{E}}_{a}(\mathbf{x})\right|^{2}$ is a normalizing factor, with $\hat{E}_{2}(\mathbf{x})$ the (normalized) electric field for mode $a$. 
From the gBL theory, one may decompose the total fluctuation spectrum $|\bar{\phi}|^{2}(a)$ into a sum over the portions $|\overline{\bar{\phi}}|^{2}(a, 2)$ driven by each species 2 , [cf. Eq.(24)]

$$
|\overline{\bar{\phi}}|^{2}(a)=\sum_{2}|\overline{\bar{\phi}}|^{2}(a, 2)
$$

Using Eq.(4) in Eq.(3), we may correspondingly decompose $\dot{W}_{0}^{q l}(1)$ :

$$
\dot{W}_{0}^{q l}(1)=\sum_{2} \dot{W}_{0}^{q l}(1 \mid 2)
$$

where

$$
\dot{W}_{0}^{q l}(1 \mid 2)=2 \sum_{a} \omega_{a} \frac{\hat{N}_{a} \Delta_{a}^{\prime \prime}\left(\omega_{a} \mid 1\right)}{4 \pi}|\overline{\bar{\phi}}|^{2}(a, 2)
$$

The gBL expression for the work done on species 1 may then be expressed analogously to Eq.(5), with the relation between $\dot{W}_{0}(1 \mid 2)$ in the two theories simply given by

$$
\dot{W}_{0}^{g \theta L}(1 \mid 2)=\dot{W}_{0}^{q l}(1 \mid 2)-\dot{W}_{0}^{q l}(2 \mid 1)
$$

The difference between the gBL and ql predictions for energy exchange is seen to come from the second term on the right side of Eq.(7), which arises from the dynamic friction $F$, absent in ql theory. From the antisymmetry of $\dot{W}_{0}^{g B L}$ in Eq.(7) it is apparent that $\dot{W}_{0}^{g B L}(1 \mid 2)+\dot{W}_{0}^{g B L}(2 \mid 1)=0$, i.e., that work done by species 2 on species 1 is minus that done by 1 on 2 , and that therefore in the gBL theory interspecies energy exchange conserves particle energy:

$$
\sum_{1} \dot{W}_{0}^{g B L}(1) \equiv \sum_{1,2} W_{0}^{g B L}(1 \mid 2)=0
$$

In ql theory, under the quasineutrality approximation, one finds an expression which looks analogous, ${ }^{8}$ but whose physical content is rather different, since in that theory there is an additional subsystem which may gain 
or lose energy, namely the waves:

$$
\sum_{1} \dot{W}^{q l}(1)=\sum_{1} \dot{W}_{0}^{q l}(1)+\sum_{1} \dot{W}_{2}^{q l}(1)=0
$$

The term $\sum_{1} \dot{W}_{2}^{q l}(1)$ here is the time derivative of the wave energy $W_{w}=$ $\sum_{a} W_{a}$. (If quasineutrality is not assumed, the field contribution to $W_{w}$ must also appear here, making more explicit that wave energy is necessary for energy conservation in ql theory.) Using $\dot{W}_{w}^{q l}=\sum_{a} 2 \gamma_{a} W_{a}$, the standard expressions for the mode growth rate $\gamma_{a} \equiv-\nu_{a}=-\Delta_{a}^{\prime \prime} / \bar{\Delta}_{a}$ (where $\left.\bar{\Delta}_{a} \equiv \partial \Delta_{a} /\left.\partial \omega\right|_{\omega_{a}}\right)$, and the energy in mode $a, W_{a}=\omega_{a} I_{a}$, with $I_{a} \equiv$ $\hat{N}_{a} \bar{\Delta}_{a}|\overline{\bar{\phi}}|^{2}(a) /(4 \pi)$ the wave action, ons finds

$$
\dot{W}_{w}^{q l}=\sum_{1} \dot{W}_{2}(1)=-2 \sum_{a} \omega_{a} \frac{\hat{N}_{a} \Delta_{a}^{\prime \prime}\left(\omega_{a}\right)}{4 \pi}|\overline{\bar{\phi}}|^{2}(a) .
$$

Using this and Eq.(3) yields Eq.(9).

On the other hand, in the gBL theory $\dot{W}_{2}=0=\dot{W}_{w}$, since the wave spectrum is at steady state. However, summing Eq.(7) over species 2 to assess the significance of the second, dynamic-friction term on the right side there, one finds

$$
\dot{W}_{0}^{g B L}(1)-\dot{W}_{0}^{q l}(1)=-2 \sum_{a} \omega_{a} \frac{\hat{N}_{a} \Delta_{a}^{\prime \prime}\left(\omega_{a}\right)}{4 \pi}|\overline{\bar{\phi}}|^{2}(a, 1) .
$$

Summing this over species 1 yields just $\dot{W}_{w}^{q l}$ in the quasilinear equation Eq.(10). That is, as indicated in Sec. I, the dynamic friction term in the gBL theory assumes the role played by the wave subsystem in preserving the conservation laws.

Eq.(9) may be written $\dot{W}_{p}^{q l}+\dot{W}_{w}^{q l} \equiv \sum_{1} \dot{W}_{0}^{q l}(1)+\sum_{a} \dot{W}_{a}^{q l}=0$. Thus, as noted in the Introduction, as opposed to the gBL law (8) for energy conservation, the ql law must include the rate of change $\dot{W}_{w}$ of wave energy 
in order to conserve energy. As may be readily shown from the expressions introduced in Sec. III, a similar statement holds for conservation of angular momentum $p_{\zeta}$. For the gBL theory one has, analogous to Eq.(8),

$$
\dot{P}_{p}^{g B L} \equiv \sum_{1} \dot{P}_{0}^{g B L}(1)=\sum_{1,2} \dot{P}_{0}^{g B L}(1 \mid 2)=0
$$

from which follows the property of intrinsic ambipolarity ${ }^{6}$

$$
\sum_{1} e_{1} \Gamma(1)=\sum_{1,2} e_{1} \Gamma(1 \mid 2)=0
$$

where $\dot{P}_{0}(1) \equiv \int d^{6} z p_{\zeta} \partial_{t} f_{0}(1)$ is the irreversible rate of change of angular momentum of species 1 , and $\Gamma(1)$ is the corresponding radial particle flux. In contrast, in ql theory, while total particle angular momentum is conserved within the quasineutral approximation, $\sum_{1} \dot{p}^{q l}(1) \equiv \sum_{1} \int d^{6} z_{1} p_{\zeta} \partial_{t} f(1)=0$, the reversible part of this is the wave momentum $\dot{P}_{w}=\sum_{a} \dot{P}_{a}$, with $P_{a}=$ $n I_{a}$. Thus:

$$
\dot{P}_{p}^{q l}+\dot{P}_{w}^{q l} \equiv \sum_{1} \dot{P}_{0}^{q l}(1)+\sum_{a} \dot{P}_{a}^{q l}=0,
$$

analogous to Eq.(9). Thus, ambipolarity of the irreversible particle fluxes (which are what are the usual quantity of interest in transport calculations) does not hold within ql theory, unless all modes have $\gamma_{a}=0$.

For the corresponding $\mathrm{H}$-theorems in the two theories, however, the same structural comparison does not hold. Using Eq.(16), and Eq.(17) or Eq.(18) in the usual definitions $\dot{S}_{p}=\sum_{1} \dot{S}(1)=-\sum_{1} d / d t \int d^{6} \tilde{z}_{1} f_{0}(1) \ln f_{0}(1)$ for the particle contribution to $\dot{S}$, and $\dot{S}_{w}=\sum_{a} \dot{S}_{a}=d / d t \sum_{a} \ln I_{a}$ for the wave contribution, $\dot{S}_{p}$ in both the $\mathrm{gBL}^{5,6}$ and ql theories are positive definite expressions, $\dot{S}_{w}=0$ in the gBL theory, but $\dot{S}_{w}=\sum_{a} 2 \gamma_{a}$ in ql theory has indefinite sign. Thus, while the $\mathrm{gBL}$ H-theorem has a form parallelling that 
of the gBL conservation laws, a ql H-theorem exists only if $\dot{S}_{w}^{q l}$ is dropped. As indicated in Sec. I, this peculiarity of the form of the ql H-theorem arises from the fact that the waves in ql theory are not in equilibrium, and moreover that their time evolution involves no statistical mixing, so that one should not expect their entropy to increase, in general.

\section{Formal Calculation}

We now introduce the necessary formal machinery, and develop the theoretical basis for the relations quoted in the previous section. and further results yet to be obtained. Our discussion of the action-angle formalism and the expressions for the $\mathrm{ql}$ and gBL theory within it is intended mainly to adequately define quantities in the formallism. The reader is referred to earlier work for elaboration in the development of the action- ingle formalism and $\mathrm{ql}$ theory, ${ }^{11}$ tha $\mathrm{gBL}$ collision operator, ${ }^{5,12}$ and for the development from this operator of the gBL transport theory. ${ }^{6,7}$

The essence of the action-angle formalism as originated in Ref. 11 is choosing a coordinate system to make the mathematical manipulations needed in solving the Vlasov-Maxwell system as simple and general as possible. For particles, this means the reparametrization of the phase point $z$ of a particle from the more directly physical set $(\mathbf{r}, \mathbf{p})$ of real-space position $\mathbf{r}$ and its conjugate momentum $\mathbf{p}$ to the set $\mathbf{J} \equiv\left(J_{1}, J_{2}, J_{3}\right)$ of the invariant actions of the unperturbed moticin, and their conjugate angles $\theta \equiv\left(\theta_{1}, \theta_{2}, \theta_{3}\right)$. (For unmagnetized plasmas, this transformation is just the identity map.) The unperturbed Hamiltonian $H_{0}$ is then independent of $\theta$, 
$H(z, t)=H_{0}(\mathbf{J})+h(\boldsymbol{\theta}, \mathbf{J}, t)$, simplifying Hamilton's equations:

$$
\begin{aligned}
& \dot{\boldsymbol{\theta}}=\partial_{\mathbf{J}} H=\Omega(\mathbf{J})+\partial_{\mathbf{J}} h, \\
& \dot{\mathbf{J}}=-\hat{\sigma}_{\mathrm{g}} h(z, t)=-i \sum_{\mathbf{l}} \mathrm{l} h(\mathbf{l}, \mathbf{J}, t) \exp (i \mathbf{l} \cdot \boldsymbol{\theta}),
\end{aligned}
$$

where $\partial_{\mathbf{J}} \equiv \partial / \partial \mathbf{J}$ denates a gradient in $\mathbf{J}$ space (and similarly for $\partial_{\theta}$ ), $\boldsymbol{\Omega}(\mathbf{J}) \equiv \partial_{\mathbf{J}} H_{0} \equiv\left(\Omega_{1}, \Omega_{2}, \Omega_{3}\right)$ is the unperturbed rate of change of $\theta$, and $1 \equiv\left(l_{1}, l_{2}, l_{3}\right)$ is a three-component vector index, specifying the Fourier harmonic. The Fourier amplitudes $h(\mathbf{l}, \mathbf{J})$ of the perturbing Hamiltonian $h(z, t)$ are the "coupling coefficients," which play a central role in the theory. As exemplified in Eq.(15), Fourier transforming with respect to $\theta$ converts $\partial_{\boldsymbol{\theta}}$ into the algebraic $i l$, and so makes derivatives $g_{0}^{-1}$ (or integrations) along unperturbed particle orbits simple, $g_{0}^{-1} \rightarrow i(1 \cdot \Omega-\omega)$, permitting a ready perturbative solution of the Vlasov equation, just as in the unmagnetized case.

For waves, the 'right' choice of representation employed in Ref. 11 is expressing fields as a sum over the plasma normal modes $a$. This permits a formal solution of the Maxwell equations, making the results of the formal. ism valid for fields in fully inhomogeneous geometries. In the simplest case. viz., a homogeneous, unmagnetized plasma, the spatial dependence of the natural basis set for both particles and waves is the same, $\sim \exp i \mathbf{k} \cdot \mathbf{r}$. This degeneracy makes the coupling coefficients $h\left(\mathrm{l}_{1}, \mathrm{~J}_{1} \mid a\right)$ of eigenmode $a-\mathrm{k}$ especially simple, $h(\mathbf{l}, \mathrm{J} \mid a-\mathbf{k}) \propto \delta(\mathbf{l}-\mathbf{k})$, causing the $\mathrm{gBL}$ operator [given by the right side of Eq.(16) and Eq.(18)] to reduce to the standard BL operator. 5

With these definitions, the equation for the evolution of $f_{0}(1)$ within 
both the ql and gBL theories is

$$
\partial_{t} f_{0}(1)=-\partial_{\mathbf{J}_{1}} \cdot \Gamma(1)
$$

For ql theory one has ${ }^{11}$

$$
\begin{aligned}
-\Gamma(1) & =D^{q l}(1) \cdot \partial_{\mathrm{J}_{1}} f_{0}(1) \\
& \equiv \sum_{a} \sum_{\mathbf{l}_{1}} 2 \pi \delta\left(\mathbf{l}_{1} \cdot \Omega_{1}-\omega_{a}\right)\left|h\left(\mathbf{l}_{1} \mid a\right)\right|^{2} \mathbf{l}_{1} \mathbf{l}_{1} \cdot \partial_{\mathrm{J}_{1}} f_{0}(1),
\end{aligned}
$$

where $\Omega_{1} \equiv \Omega\left(\mathrm{J}_{1}\right)$, and we denote $h(\mathrm{l}, \mathrm{J} \mid a)$ by the shorthand $h(\mathrm{l} \mid a)$. In the gBL theory, one finds instead $\Gamma(1)=-\mathbf{D}(1) \cdot \partial_{\mathbf{J}_{1}} f_{0}(1)+\mathbf{F}(1) f_{0}(1)$, where both $\mathrm{D}(1)$ and $\mathbf{F}(1)$ individually, and so $\Gamma(1)$ as a whole, may be written as a sum over contributions from interactions of species 1 with species 2 , e.g. $\Gamma(1)=\sum_{2} \Gamma(1 \mid 2)$. (Henceforth, unless unclear from context, we suppress the superscript on gBL quantities.) $\Gamma(1 \mid 2)$ is given by

$$
-\Gamma(1 \mid 2)=\sum_{\mathbf{l}_{1}, \mathrm{l}_{2}} \int d^{6} z_{2} Q(1,2) \mathbf{l}_{1}\left(\mathbf{l}_{1} \cdot \partial_{\mathbf{J}_{1}}-\mathbf{l}_{2} \cdot \partial_{\mathbf{J}_{2}}\right) f_{0}(1) f_{0}(2)
$$

with kørnel

$$
Q(1,2)=Q(2,1)=\sum_{a} 2 \pi \delta\left(1_{1} \cdot \Omega_{1}-l_{2} \cdot \Omega_{2}\right)|4 \pi \alpha(1,2 \mid a)|^{2}
$$

and with $\alpha(1,2 \mid a) \equiv \hat{h}\left(\mathrm{l}_{1} \mid a\right) \hat{h}^{*}\left(\mathrm{l}_{2} \mid a\right) /\left.\left[\hat{N}_{a} \Delta_{a}(\omega)\right]\right|_{\omega=l_{1} \cdot \Omega_{1}}$ measuring the effectiveness of mode $a$ in coupling particles 1 and 2. Here, $\hat{h}(. . \mid a)$ is the coupling coefficient due to the normalized fields $\hat{\mathbf{E}}_{a}(\mathbf{x})$, so that $\alpha(1,2 \mid a)$ as defined is independent of the particular normalization chosen. Consistent with the results quoted in Sec. II, we choose $\hat{h}$ so that the mode amplitudes $\overline{\bar{\phi}}$ appear explicitly, $h\left(\mathbf{l}_{1} \mid a\right)=\overline{\bar{\phi}}(a) \hat{h}\left(\mathbf{l}_{1} \mid a\right)=\overline{\bar{\phi}}(a) e_{1} \hat{\phi}\left(\mathbf{l}_{1} \mid a\right)$. The diffusive portion is the term in $l_{1} l_{1}$ in Eq.(18), and the frictional portion is the term in $l_{1} l_{2}$. [In Eqs.(17) and (19), a factor of 2 , accompanying the $\pi \delta()$ there, was omitted 
in Refs.5 and 6.] The general formalism is fully electromagnetic. As suggested by the forms chosen above for $h$, henceforth, for simplicity, we shall restrict ourselves to electrostatic fluctuations, $h\left(z_{1}, t\right)=e_{1} \phi\left[\mathbf{r}\left(z_{1}\right), t\right]$.

Comparing $\mathbf{D}^{q /}(1)$ in (17) with the $g B L$ expression for $D(1 / 2)$ or $D(1)$ read from Eqs.(18) and (19), one notes the replacements $\omega_{a}$ by $\omega_{2} \equiv l_{2} \cdot \Omega_{2}$ in the $\delta$-function, $\sum_{a} \rightarrow \sum_{a, l_{2}} \int d^{6} z_{2} f_{0}(2)$ in the summations, and $h\left(l_{1} \mid a\right)-$ $4 \pi \alpha(1,2 \mid a)$ for the cospling amplitudes. Thus, in the gBL theory, each increment $d^{6} z_{2}$ of phase space of species 2 contributes to the overall spectrum appearing in $D^{q l}$, driving fluctuations over a range of frequencies $\omega_{2}\left(l_{2}, J_{2}\right)$. We make this more explicit in Eq.(24).

Finally, from the action-angle expression for the nonlocal susceptibility tensor $^{11} \Delta\left(\mathbf{x}, \mathbf{x}^{\prime}, \omega \mid 1\right)$ for species 1 , one has ${ }^{5}$

$$
\begin{aligned}
\hat{N}_{a} \Delta_{a}^{\prime \prime}(\omega \mid 1) & =\int d \mathbf{x} d \mathbf{x}^{\prime} \hat{\mathbf{E}}_{a}^{*}(\mathbf{x}) \cdot \Delta^{\prime \prime}\left(\mathbf{x}, \mathbf{x}^{\prime}, \omega \mid 1\right) \cdot \hat{\mathbf{E}}_{a}\left(\mathbf{x}^{\prime}\right) \\
& =-4 \pi \sum_{\mathrm{l}_{1}} \int d^{6} z_{1} \pi \delta\left(\mathbf{l}_{1} \cdot \Omega_{1}-\omega\right)\left|\hat{h}\left(\mathbf{l}_{1} \mid a\right)\right|^{2} \mathbf{l}_{1} \cdot \partial_{\mathbf{J}_{1}} f_{0}(1)
\end{aligned}
$$

Having assembled these earlier results, we now turn to consideration of $\dot{W}_{0}$. Using Eq.(16) and integrating by parts with respect to $J_{1}$, one finds, for both theories,

$$
\dot{W}_{0}(1) \equiv \int d^{6} z_{1} H_{0}(1) \partial_{t} f_{0}(1)=\int d^{6} z_{1} \Omega_{1} \cdot \Gamma(1) .
$$

Using the ql expression (17) in Eq.(21), one finds

$$
\dot{W}_{0}^{q l}(1)=2 \sum_{a} \omega_{a}(4 \pi)^{-1}\left[-4 \pi \sum_{\mathrm{l}_{1}} \int d^{6} z_{1} \pi \delta\left(\omega_{1}-\omega_{a}\right)\left|h\left(\mathrm{l}_{1} \mid a\right)\right|^{2} \mathrm{l}_{1} \cdot \partial_{\mathrm{J}_{1}} f_{0}(1)\right]
$$

where $\omega_{1} \equiv l_{1} \cdot \Omega_{1}$. Noting from Eq.(20) that the term in square brackets here is $N_{a} \Delta_{a}^{\prime \prime}\left(\omega_{a} \mid 1\right) \equiv \hat{N}_{a} \Delta_{a}^{\prime \prime}\left(\omega_{a} \mid 1\right)|\overline{\bar{\phi}}|^{2}(a)$, demonstrates expression (3). 
Similarly, for the gBL counterpart, using Eqs.(18) and (19) in Eq.(21), we obtain

$$
\begin{aligned}
\dot{W}_{0}(1 \mid 2)= & -2 \sum_{a} \sum_{l_{1}, l_{2}} \int d^{6} z_{1} \int d^{6} z_{2} \omega_{1} \pi \delta\left(\omega_{1}-\omega_{2}\right)|4 \pi \alpha(1,2 \mid a)|^{2} \\
& \times\left(l_{1} \cdot \partial_{J_{1}}-l_{2} \cdot \partial_{\mathbf{J}_{2}}\right) f_{0}(1) f_{0}(2) \\
= & 2 \sum_{a} \sum_{l_{2}} \int d^{6} z_{2} f_{0}(2)\left|\frac{4 \pi \hat{h}\left(l_{2} \mid a\right)}{\hat{N}_{a} \Delta_{a}\left(\omega_{2}\right)}\right|^{2} \omega_{2} \frac{\hat{N}_{a} \Delta_{a}^{\prime \prime}\left(\omega_{2} \mid 1\right)}{4 \pi}-(1-2),
\end{aligned}
$$

where Eq.(20) has again been used, and we have made use of the fact that the $\delta$-function sets $\omega_{1}$ equal to $\omega_{2}$. The second term $(1 \rightarrow 2)$ in the final form here arises from $\Gamma_{F}$.

Comparing Eq.(22) with the ql form (3), one notes that the result ( 7 ) has almost been reached, but not quite yet, because the $\omega_{a}$ dependence in Eq.(3) has been replaced, as noted following Eq.(19), by a sum $\sum_{\mathrm{l}_{2}} \int d^{6} z_{2}$ over contributions to fluctuations driven at frequency $\omega_{2}=l_{2} \cdot \Omega_{2}$. If one further assumes that the dielectric response in the denominator in (22) produces a spectrum sharply peaked about $\omega_{2}=\omega_{a}$, as one expects for a weakly coupled thermal plasma, or one supporting weak turbulence, writing

$$
\left|\Delta_{a}\left(\omega_{2}\right)\right|^{-2} \simeq 1 /\left(\left|\bar{\Delta}_{a}\right|^{2}\left|\nu_{a}\right|\right) \pi \delta\left(\omega_{2}-\omega_{a}\right),
$$

Eq.(22) yields ( 7 ), with the identification

$$
\begin{aligned}
|\bar{\phi}|^{2}(a, 2) & \equiv \sum_{l_{2}} \int d^{6} z_{2} f_{0}(2)\left|\frac{4 \pi \hat{h}\left(l_{2} \mid a\right)}{\hat{N}_{a} \Delta_{a}\left(\omega_{2}\right)}\right|^{2} \\
& \simeq \sum_{l_{2}} \int d^{6} z_{2} f_{0}(2)\left|\frac{4 \pi \hat{h}\left(l_{2} \mid a\right)}{\hat{N}_{a} \bar{\Delta}_{a}}\right|^{2} \frac{\pi}{\left|\nu_{a}\right|} \delta\left(\omega_{2}-\omega_{a}\right) .
\end{aligned}
$$

With the same identification, $\mathrm{D}^{g B L}(1)$ from Eq.(18) and (19) reduces to $D^{a l}(1)$ from Eq.(17). Neither the ql nor gBL theories specify the magnitude $|\overline{\bar{\phi}}(a)|^{2}$ of the overall spectrum, which may be chosen to correspond to 
experimental observations. In conjunction with Eq.(4), Eq.(24) provides a prescription for computing the relative sizes of the contributions $|\overline{\bar{\phi}}(a, 2)|^{2}$ to $|\overline{\bar{\phi}}(a)|^{2}$, thus leaving only the single set of amplitudes $|\overline{\bar{\phi}}(a)|^{2}$ as external to the theory.

With the narrow-width approximation (23) for the spectrum made in the second form of Eq.(24), $\dot{W}_{0}(1 \mid 2)$ in Eq.(22) acquires a noteworthy symmetric character, reflecting the symmetry between the emittor and absorber of fluctuations in the $\mathrm{gBL}$ (or BL) collision operator:

$$
\begin{aligned}
\dot{W}_{0}(1 \mid 2)= & 2 \sum_{a} \frac{\omega_{a}}{\left|\hat{N}_{a} \bar{\Delta}_{a}\right|^{2}\left|\nu_{a}\right|}\left\langle\left\langle\pi \delta\left(w_{2}-w_{a}\right) 4 \pi\left|\hat{h}\left(l_{2} \mid a\right)\right|^{2}\right\rangle\right\rangle_{2} \\
& \times\left\langle\left\langle-\pi \delta\left(w_{1}-w_{a}\right) 4 \pi\left|\hat{h}\left(l_{1} \mid a\right)\right|^{2} l_{1} \cdot \partial_{\mathrm{J}_{1}} \ln f_{0}(1)\right\}\right\rangle_{1}-(1-2),
\end{aligned}
$$

where $\langle\langle.\rangle.\rangle \equiv \sum_{1} \int d^{6} z f_{0} \ldots$ In the factor $\langle\langle. .\rangle\rangle_{1}=\hat{N}_{a} \Delta_{a}^{\prime \prime}(\mid 1)$ here one recog. nizes the response of the scattered species in (20), while in the quite similar factor $\langle\langle. .\rangle\rangle_{2} \equiv \psi(a, 2)$ one sees the spectrum $|\overline{\bar{\phi}}|^{2}(a, 2)=4 \pi /\left(\left|\hat{N}_{a} \vec{\Delta}_{a}\right|^{2}\left|\nu_{a}\right|\right) \psi$ from the scattering species, given in Eq.(24).

\section{Application of the General Formalism}

\section{A. Specialization to Toroidal Geometry}

In the abstract forms we have dealt with thus far, the formalism applies equally well to any geometry where the unperturbed motion is integrable, including uniform, unmagnetized plasmas, magnetized slabs, and axisymmetric tori. In the last case, of principal interest here, the specialization is $\left(J_{1}, J_{2}, J_{3}\right) \rightarrow\left(J_{g}, J_{b}, J_{\zeta} \equiv p_{\zeta}\right)$, with $J_{g}$ the gyroaction (equal to $M c / \epsilon$ times the usual magnetic moment $\mu$ ), $J_{b}$ the bounce-action, and $p_{\zeta}$, the toroidal angular momentum. ( $\zeta$ is the toroidal azimuth, which, along with 
poloidal azimuth $\theta$ and a minor-radial variable $r$, constant on a flux surface, parametrize real space.) The transformation from the mathematically convenient variables $\mathrm{J}$ to a more physical set is discussed in Refs. 11,6,13 and 14. Briefly, for magnetized plasmas and tori in particular, certain combinations of the $J_{i}$ retain a velocity-like character, while others acquire a spatial one, specifying the particle 'banana center' $r_{b}(J)$, the average minor radial value about which a particle moves in the course of a bounce (or transit) time. For example, for trapped particles, $r_{b}$ is a function of $p_{\zeta}$ alone, while $J_{b}$ is $v_{\|}$-like, and $J_{g}$ is $v_{\perp}$-like.

For definiteness, we choose for a model of the mode structure the eikonal form $^{6}$

$$
\phi(\mathbf{x} \mid a)=\bar{\phi}(r \mid a) \exp i \theta_{a}(\mathbf{x})
$$

with wave phase $\theta_{a}(\mathbf{x}) \equiv\left[\int^{\tau} d r^{\prime} k_{r}\left(r^{\prime}\right)+m \theta+n \zeta\right]$, and with modulating envelope $\bar{\phi}(r \mid a)$, localizing the mode about $r=r_{a}$, with mode width $w_{a}$. For $\bar{\phi}(r \mid a)$ we take the simplified form $\bar{\phi}(r \mid a)=\overline{\bar{\phi}}(a) s\left(w_{a} / 2, r-r_{a}\right)$, where $s(x, y)$ is a step-like localizing function, defined as

$$
s(x, y) \equiv \begin{cases}1 & (x \geq|y|) \\ 0 & (x<|y|) .\end{cases}
$$

While obviously simplified, this model captures features one expects for a set of modes comprising plasma turbulence, including a locally wavelike character, radially localized, and constituting a complete, orthogonal basis set. Within this model, one has $\dot{N}_{a}=k^{2} V_{a}$, with $V_{a} \simeq\left(2 \pi r_{a}\right)(2 \pi R) w_{a}$ the volume of the shell around $r=r_{a}$ within which mode $a$ is localized. Assuming the banana width $\rho_{b}$ of the particles is less than localization width 
$w_{a}$, the coupling coefficients for the model are given by ${ }^{6}$

$$
\begin{aligned}
& h(1 \mid a)=\bar{\phi}(a) \hat{h}(1 \mid a), \\
& \ddot{h}(1 \mid a)=e \ddot{\phi}(1 \mid a)=e s\left(w_{a} / 2, r_{b}-r_{a}\right) G(1 \mid a), \\
& G(1 \mid a)=\delta\left(l_{\zeta}-n\right) J_{l_{g}}\left(z_{g}\right) J_{l_{b}}\left(z_{b}\right) \exp -i \chi_{a} .
\end{aligned}
$$

Here, the $G(1 \mid a)$ are the "orbit-averaging" factors, measuring the fraction of time along its orbit a particle sees a contribution from mode $a$ oscillalory at $\exp i l \cdot \theta$. Since $G(1)$ appears only as $|G(1)|^{2}$ in quantities of interest here, the phase factor $\chi_{a}$ is irrelevant. And because the $G(1)$ are the Fourier components of the eikonal factor exp $i \theta_{a}$ in Eq.(26), by Parseval's theorem they satisfy the important relation $1=\sum_{1}|G(1)|^{2}$, generalizing the muchused identity for Bessel functions $1=\sum_{l} J_{l}^{2}(z)$. In Eq.(28), $l_{b}^{\prime} \equiv l_{b}-\sigma m$, $J_{l_{g}}$ arises from the integration over gyrophase $\theta_{g}$, and $J_{l_{b}^{\prime}}$ comes from the analogous integration over bounce phase $\theta_{b} . \sigma$ is a trapping-state index. equal to $0(1)$ for trapped(passing) particles, and $z_{g}=k_{\perp} \rho_{g}$. The full expression ${ }^{13,6}$ for $z_{b}$ is slightly more complicated than that for $z_{g}$, and defining it fully introduces extra notation not needed here. For turbulent modes. characterized by $k_{\|} \sim 1 / q R, k_{\perp} \sim \rho_{g i}^{-1}$, the essential physics is captured by approximating it as $z_{b} \simeq k_{\perp} \rho_{b}$.

We now use the model of Eqs. (26)-(28) in evaluating the earlier formal expressions. For example, we consider the factors $\langle(.\rangle$.$\rangle in Eq.(25). In the$ summation over 1 there, the factor $\delta\left(l_{\zeta}-n\right)$ in $G(1)$, a consequence of axisymmetry, fixes $l_{\zeta}$ to $\bar{l}_{\zeta} \equiv n$. $J_{l}(z)$ is appreciable only for $|l|<z$, and falls off rapidly for larger $l$. Thus, if we in addition restrict ourselves to modes in having frequency $\omega_{3}$ small compared with the ion gyrofrequency $\Omega_{g i}$, the resonance coridition $\omega_{a}=\omega_{2}$ imposed by the delta-function in 
$\langle(. .\rangle\rangle_{2}$ is satisfied for nonnegligible values of $J_{l_{b}^{\prime}}\left(z_{b}\right)$, hence of $G(l)$, only for $l_{g}=I_{g} \equiv 0$. This reduces the triple sum over $l$ to a single sum over $l_{b}$ or $l_{b}^{\prime}$. Additionally, for Julent modes, the range $\Delta l_{b} \sim z_{b}$ over which $J_{l_{b}^{\prime}}$ is appreciable is small compared with $m$. Thus, one may approximate $l_{b}$ by $\bar{l}_{b} \equiv \sigma m$ in the factor in $\langle\langle.\rangle$.$\rangle accompanying \delta()|\hat{h}(1 \mid a)|^{2}$, thus replacing 1 by $\bar{l} \equiv\left(\bar{l}_{g}, \bar{l}_{b}, \bar{l}_{\zeta}\right)$ there.

Now adopting the local Maxwellian form for $f_{0}$,

$$
f_{m}(\mathrm{~J}) \equiv \frac{n}{(2 \pi M T)^{3 / 2}} \exp \left[-\left(H_{0}-e \Phi\right) / T\right),
$$

where density $n$, radial potelitial $\Phi$, and temperature $T$ are functions of $r_{b}(\mathrm{~J})$, and $M$ is the particle mass, the factor $\overline{\mathrm{I}}_{1} \cdot \partial_{\mathrm{J}_{1}} \ln f_{0}(1)$ in $\langle(. .)\rangle_{1}$ is given by 6

$$
\bar{I} \cdot \partial_{\mathrm{J}} \ln f_{m}=\left(\omega_{-}^{\prime}-\bar{\complement} \cdot \Omega\right) / T,
$$

where $\omega^{f} \equiv \omega_{*}\left[1+\eta\left(u^{2}-3\right) / 2\right]$, with $\omega_{*} \equiv-k_{y} c T /\left(e B L_{n}\right)$ the diamagnetic drift frequency, $\eta \equiv d \ln T / d \ln n, u \equiv v / v_{s}$ the particle velocity, normalized to the thermal speed $v_{s}, k_{y} \equiv \dot{b} \times \hat{r} \cdot \mathbf{k}, L_{n}^{-1} \equiv-\partial \ln n_{0} / \partial r$, and $\omega_{d}=n \Omega_{\zeta}(\sigma=$ 0 ) is the toroidal drift frequency.

Using Eqs. (28) and (30), one finds

$$
\begin{aligned}
\psi(a, 2) & \equiv\langle\langle. . .\rangle\rangle_{2}=V_{a} 4 \pi e_{2}^{2} n_{2} \beta_{a}\left(\omega_{a} \mid 2\right), \\
\beta_{a}(\omega \mid 2) & \equiv \sum_{l_{b 2}}\left\langle\pi \delta\left(\omega_{2}-w\right)\left|G\left(l_{2} \mid a\right)\right|^{2} \mid\right\rangle_{i_{g 2}, I_{\zeta 2}},
\end{aligned}
$$

where $\langle..\rangle \equiv\left(V_{a} n\right)^{-1} \int_{V_{a}} d^{6} z f_{0}$ is the average over the shell $V_{a}$, with $\int_{V_{a}} d^{6} z$ the phase-space integration confined to $V_{a}$. Similarly,

$$
\begin{aligned}
\hat{N}_{a} \Delta_{a}^{\prime \prime}\left(\omega_{a} \mid 1\right) & \equiv\langle\langle. .\rangle\rangle_{1}=V_{a} 4 \pi e_{1}^{2} n_{1} / T_{1}\left[-\alpha_{a}\left(\omega_{a} \mid 1\right)\right], \\
-\alpha_{a}(\omega \mid 1) & \equiv \sum_{l_{b 1}}\left\langle\pi \delta\left(\omega_{1}-w\right)\left|G\left(l_{1} \mid a\right)\right|^{2}\left(\omega_{1}-\omega_{a 1}^{f}\right)\right\rangle_{i_{g 1}, I_{b 1}},
\end{aligned}
$$


and therefore, Eq.(25) yields

$$
\begin{aligned}
\dot{W}_{0}(1 \mid 2)= & 2 \sum_{a}\left|\frac{4 \pi e_{1} e_{2}}{\hat{N}_{a} \bar{\Delta}_{a}}\right|^{2} \frac{\omega_{a}}{\left|\nu_{a}\right|} \sum_{l_{b 1}, l_{b 2}} \int_{V_{a}} d^{6} z_{1} \int_{V_{a}} d^{6} z_{2} f_{m}(1) f_{m}(2) \times \\
& \pi \delta\left(\omega_{1}-w_{a}\right) \pi \delta\left(\omega_{2}-\omega_{a}\right)\left|G\left(l_{1} \mid a\right)\right|^{2}\left|G\left(l_{2} \mid a\right)\right|^{2}\left[\omega_{a}\left(\frac{1}{T_{1}}-\frac{1}{T_{2}}\right)-\left(\frac{\omega_{-1}^{\prime}}{T_{1}}-\frac{\omega_{-2}^{\prime}}{T_{2}}\right)\right]
\end{aligned}
$$

where use has been made of the fact that $w_{1}=w_{2}=w_{a}$, due to the $\delta$ functions.

In the homogeneous limit where $f_{m}$ becomes a true Maxwellian $f_{M}, \omega !$ becomes negligible, and Eq.(33) reduces to $\dot{W}_{0}(1 \mid 2)=\sum_{a} \omega_{a}^{2}\left(T_{1}^{-1}-T_{2}^{-1}\right)(.$.$) ,$ where (..) is positive definite, and may be read off from Eq.(33). Thus, for $T_{1}<T_{2}$, the gBL theory predicts $\dot{W}_{0}(1 \mid 2)>0$, i.e., that energy will flow 'downhill,' from species 2 to species 1, as indicated in Sec. II. As noted there, the ql prediction, obtained from Eq.(33) by dropping the two terms involving $T_{2}$, possesses no such property. We note that a similar, more general proof of this may be given, without making the narrow-width approximation (23) or the assumption of the mode model in Eqs. (26)-(28) made in this section. The more specialized version has been given to make the forms appearing more immediately resemble more conventional expressions occurring outside the action-angle formalism, for example, expression (32) for $\Delta_{a}^{\prime \prime}$.

\section{B. Application to the Trapped Electron Mode}

We now further specialize expression Eq.(25) or (33) to considering the collisionless TEM (CTEM). This introduces additional simplifications in the expressions going into $\dot{W}_{0}$, serving as a illustrative example by which one may more concretely compare the predictions of the $\mathrm{ql}$ and gBL theories.

Expression (25) thus far explicitly involves $\Delta_{a}^{\prime \prime}(\mid 1)$, through the factor 
$\langle(. .)\rangle_{1}$, and also implicitly calls for the full susceptibility $\Delta_{a}(\mid 1)$, through $\omega_{a} . \Delta_{a}(\mid 1)$ is given simply by ${ }^{11,5}$ making the replacement $-\pi \delta\left(\omega-\omega_{1}\right) \rightarrow$ $\left(\omega-\omega_{1}\right)^{-1}$ in expression $(20)$ for $\Delta_{a}^{\prime \prime}(\mid 1)$ :

$$
\hat{N}_{a} \Delta_{a}(\omega \mid 1)=4 \pi \sum_{l_{1}} \int d^{6} z_{1} \frac{1}{\omega-\omega_{1}}\left|\hat{h}\left(l_{1} \mid a\right)\right|^{2} l_{1} \cdot \partial_{J_{1}} f_{0}(1) .
$$

Following the same specializing steps leading from Eq. $(20)$ to $\hat{N}_{a} \Delta_{a}^{\prime \prime}=\langle\langle. . .\rangle\rangle_{1}$ in Eq. (32), one finds $\Delta_{a}(\omega \mid 1)=\left(k \lambda_{D_{1}}\right)^{-2} g_{a}(\omega \mid 1)$, with $\lambda_{D_{1}} \equiv\left(T_{1} / 4 \pi n_{1} e_{1}^{2}\right)^{1 / 2}$ the Debye length of species 1 , and

$$
g_{a}(\omega \mid 1)=1-\sum_{l_{b}^{\prime}}\left\langle\frac{\omega-\omega_{-1}^{\prime}}{\omega-\sigma k_{\|} v_{\|}-(1-\sigma) \omega_{d 1}-l_{b}^{\prime} \Omega_{b 1}} J_{0}^{2}\left(z_{g}\right) J_{l_{b}}^{2}\left(z_{b}\right)\right\rangle .
$$

Here, we have converted to the more familia:, physical variables using $\omega_{1} \simeq$ $\sigma k_{\|} \bar{v}_{\|}+(1-\sigma) \omega_{d 1}+l_{b}^{\prime} \Omega_{b 1}$, with $\overline{v_{\|}}$the bounce-averaged parallel velocity, and $\omega_{d} \equiv n \Omega_{\zeta}(\sigma=0)$ the toroidal drift frequency.

We adopt the standard treatment for electrons. One has $z_{g, b} \rightarrow 0$, so that $J^{2} J^{2} \rightarrow \delta\left(l_{b}^{\prime}\right)$ in Eq.(35). Taking the usual ordering $\xi_{e} \equiv \omega /\left|k_{\|} v_{e}\right| \ll 1$, the nonadiabatic portion of the electron response is dominated by trapped particles:

$$
g_{a}(\omega \mid e) \simeq 1-F_{t}\left(\frac{\omega-\omega_{\text {ee }}^{\prime}}{\omega-\omega_{d e}}\right)_{t} \simeq 1-i \alpha_{a}(\omega \mid e),
$$

where $F_{t} \sim \epsilon^{1 / 2}$ is the fraction of trapped particles, $\langle. .\rangle_{t}$ is the average over the trapped portion of the distribution, and

$$
\begin{aligned}
\alpha_{a}(\omega \mid e) & =-F_{t}\left\langle\left(\omega-\omega_{-e}^{J}\right) \pi \delta\left(\omega-\omega_{d e}\right)\right\rangle_{t} \\
& \simeq 2 \sqrt{\pi} F_{l}\left(1-\frac{\omega}{\omega_{* e}}\right) \frac{\omega_{n e}}{\left|\bar{\omega}_{d e}\right|} \vec{E}^{1 / 2} e^{-E_{\mid E}=\omega / \bar{\omega}_{d e}}
\end{aligned}
$$

Here, $\bar{E} \equiv u^{2} / 2 \equiv E / T, \omega_{d e}=\bar{\omega}_{d e} \bar{E}$, and for simplicity we have taken $\eta_{e}=0$ in the second form. Similarly, $\beta_{a}(\mid e)$ in Eq.(31) becomes

$$
\beta_{1}(\omega \mid e)=F_{t}\left(\pi \delta\left(\omega-\omega_{d e}\right)\right\rangle_{t}
$$




$$
\left.\simeq F_{t} \frac{2 \sqrt{\pi}}{\left|\bar{\omega}_{d e}\right|} E^{1 / 2} e^{-E}\right|_{E=\omega / \bar{\omega}_{d e}} .
$$

For lons, the standard analytic ordering is that $\xi \equiv \xi_{i} \equiv \omega /\left|k_{\|} v_{i}\right| \sim$ $\omega / \Omega_{b i} \gg 1$, and in this case, the denominator in Eq.(35) does not change greatly over the $l_{b}^{\prime}$ range over which $J_{l_{b}^{\prime}}$ is appreciable, allowing one to perform the summation over $l_{b}^{\prime}$ using the identity $1=\sum_{l} J_{l}^{2}(z)$. This eliminates the factor $J_{l_{b}^{\prime}}^{2}$, leaving only $J_{0}^{2}\left(z_{g}\right)$. The dominant contribution for ions comes from passing particles:

$$
\begin{aligned}
g_{a}(\omega \mid i) & =1-F_{p}\left\langle\frac{\omega-\omega_{u_{i}}^{f}}{\omega-k_{\|\bar{v}\|}} J_{0}^{2}\left(z_{g}\right)\right\rangle_{p} \\
& \simeq 1-F_{p}\left(1-\frac{\omega_{u i}}{\omega}\right)[1-W(\xi)] \Lambda_{0}\left(b_{g}\right)
\end{aligned}
$$

with $F_{p} \simeq 1$ the fraction of passing particles, $\langle. .\rangle_{p}$ the average over the passing portion of the distribution, $b_{g} \equiv k_{\perp}^{2} \rho_{g i}^{2}, \Lambda_{0} \equiv\left\langle J_{0}^{2}\left(z_{g}\right)\right\rangle=I_{0}\left(b_{g}\right) e^{-b_{g}}$. and $[1-W(\xi)] \equiv \xi\left\langle\left(\xi-u_{\|}\right)^{-1}\right\rangle$. In the second form, we have assumed $\eta_{i}=0$ for simplicity. One has the limit $[1-W(\xi \gg 1)] \simeq 1+\xi^{-2}-$ $i W_{I}(\xi)$, with $W_{I}(\xi)=\sqrt{\pi / 2} \xi \exp -\xi^{2} / 2$. The term $\xi^{-2}$, important for the ion temperature gradient mode, to which most of the formulae developed here also apply, may be neglected for the TEM. This yields

$$
g_{a}(\omega \mid i) \simeq 1-F_{p}\left(1-\frac{\omega_{a i}}{\omega}\right) \Lambda_{0}-i \alpha_{a}(\omega \mid a)
$$

where

$$
\begin{aligned}
\alpha_{u}(\omega \mid i) & =-F_{p}\left\langle\left(\omega-\omega_{-i}^{f}\right) \pi \delta\left(\omega-k_{\|} \overline{v_{\|}}\right) J_{0}^{2}\left(z_{g}\right)\right\rangle_{p} \\
& \simeq \sqrt{\pi / 2} F_{p}\left(\frac{\omega_{-i}}{\omega}-1\right) \Lambda_{0} \xi \exp -\xi^{2} / 2,
\end{aligned}
$$

quite similar in form to $\alpha_{a}(\mid e)$, but, roughly speaking, with $\xi^{2} / 2$ replacing $\bar{E}$ there. Similarly, $\beta_{a}(\mid i)$ is given by

$$
\beta_{a}(\omega \mid i)=F_{p}\left(\pi \delta\left(\omega-k_{\|} \bar{v}_{\|}\right) J_{0}^{2}\left(z_{g}\right)\right\rangle_{p}
$$




$$
\simeq F_{p} \frac{\sqrt{\pi / 2}}{\left|k_{\|} v_{i}\right|} \Lambda_{0} \exp -\xi^{2} / 2 .
$$

From quasineutrality, the TEM dispersion relation is then

$$
\begin{aligned}
0 & =g_{a}(\omega \mid e)+\tau g_{a}(\omega \mid i) \\
& =\left(1-i \alpha_{a}\right)+\tau\left(1-\Lambda_{0}\right)-\Lambda_{0} \frac{\omega_{\omega e}}{\omega}
\end{aligned}
$$

where $\tau \equiv T_{e} / T_{i}, \alpha_{a} \equiv \alpha_{a}(\mid e)+\tau \alpha_{a}(\mid i)$, we have set $F_{p}$ to 1 , and used $\omega_{* e}=-\tau \omega_{* i}$. Solving this for the real and imaginary parts of $\omega=\omega_{a}+i \gamma_{a}$, one finds

$$
\omega_{a}=\omega_{* e} \Lambda_{0} /\left[1+\tau\left(1-\Lambda_{0}\right)\right], \gamma_{a}=\alpha \omega_{* e} \Lambda_{0} /\left[1+\tau\left(1-\Lambda_{0}\right)\right]^{2}
$$

With explicit expressions for all necessary quantities, we can now write out $\dot{W}_{0}$ for both the gBL and ql theories, for the TEM mode. Reinstating the ' $\mathrm{gBL}$ ' and 'ql' superscripts, for a 2-species plasma, for which $\dot{W}_{0}^{g B L}(1)=$ $\dot{W}_{0}^{g B L}(1 \mid 2)$, Eq. (25) or (33) yields

$$
\begin{aligned}
\dot{W}_{0}^{g B L}(1) & =\dot{W}_{0}^{q l}(1 \mid 2)-\dot{W}_{0}^{q l}(2 \mid 1) \\
& =2 \sum_{a} \omega_{a}\left[\frac{|\overline{\bar{\phi}}|^{2}(a, 2)}{4 \pi} \hat{N}_{a} \Delta_{a}^{\prime \prime}(\mid 1)-\frac{|\overline{\bar{\phi}}|^{2}(a, 1)}{4 \pi} \hat{N}_{a} \Delta_{a}^{\prime \prime}(\mid 2)\right], \\
{[\ldots] } & \simeq\left|\frac{4 \pi e_{1} e_{2}}{\hat{N}_{a} \bar{\Delta}_{a}}\right|^{2} \frac{1}{\left|\nu_{a}\right|} n_{1} n_{2} \beta_{a}(\mid 1) \beta_{a}(\mid 2)\left(\frac{\omega_{a}-\omega_{* 1}}{T_{1}}-\frac{\omega_{a}-\omega_{* 2}}{T_{2}}\right),
\end{aligned}
$$

where we have used Eqs. (31),(32), and $-\alpha_{a}() \simeq\left(\omega_{a}-\omega_{*}\right) \beta_{a}()$, following from the final forms in Eqs. (37),(38),(41), and (42). This may be compared with the ql form,

$$
\begin{aligned}
\dot{W}_{0}^{q l}(1) & =\dot{W}_{0}^{q l}(1 \mid 2)+\dot{W}_{0}^{q l}(1 \mid 1) \\
& =2 \sum_{a} \omega_{a}\left[\frac{|\bar{\phi}|^{2}(a, 2)}{4 \pi} \dot{N}_{a} \Delta_{a}^{\prime \prime}(\mid 1)+\frac{|\overline{\bar{\phi}}|^{2}(a, 1)}{4 \pi} \hat{N}_{a} \Delta_{a}^{\prime \prime}(\mid 1)\right]
\end{aligned}
$$


One notes that $\dot{W}_{0}^{g B L}$ and $\dot{W}_{0}^{g l}$ share a common first term, to which we refer by its argument (1|2), and each has a different second term, (2|1) for the $\dot{W}_{0}^{g B L}$, and $(1 \mid 1)$ for $\dot{W}_{0}^{q l}$. Thus, which theory predicts a larger $\dot{W}_{0}$ depends upon the relative sizes of these three terms. From Eqs. (37),(38),(41), and (42), these stand in the proportion

$$
(1 \mid 2):(1 \mid 1):(2 \mid 1):: 1: \frac{\beta_{a}(1)}{\beta_{a}(2)}: \frac{T_{1}}{T_{2}} \frac{\omega_{a}-\omega_{u 2}}{\omega_{a}-\omega_{m 1}}
$$

The two ratios represented here are independent, so that $\dot{W}_{0}^{g B L}$ in general may be larger than, comparable to, or smaller than $\dot{W}_{c}^{q l}$, depending upon parameters. As one sees from Eqs. (38) and (42), the factors $\beta_{a}$ in Eq.(45) are essentially a measure of how many particles of that species resonate with mode $a$, thus controlling how large the fluctuations of $a$ driven by that species are, as well as (through the close relation of $\alpha_{a}$ to $\beta_{a}$ ) how readily that species absorbs those fluctuations. Thus, for example, if parameters were such that many electrons could resonate with the TEM spectrum, but very few ions $\left[\beta_{a}(\mid e) \gg \beta_{a}(\mid i)\right]$, one would have the ordering $\dot{W}_{0}^{q l}(e) \simeq$ $\dot{W}_{0}^{q l}(e \mid e) \gg \dot{W}_{0}^{g B L}(e)=-\dot{W}_{0}^{g B L}(i) \sim \dot{W}_{0}^{q l}(e \mid i) \sim \dot{W}_{0}^{q l}(i \mid e) \sim \dot{W}_{0}^{q l}(i)$. For similar reasons, an analogous situation holds for the orderings of particle fluxes $\Gamma(1 \mid 2)$ in neoclassical (' $n c$ ') theory (or its gBL extension to turbulent transport $\left.t^{6, \pi}\right)$, in which the momentum-preserving dynamic friction term in the BL collision operator is kept, versus a similar, but cruder test-particle ('tp') calculation, where $\mathbf{F}$ is dropped. The corresponding ordering there is $\Gamma^{t p}(i) \simeq \Gamma^{t p}(i \mid i) \gg \Gamma^{n c}(i)=\Gamma^{n c}(e) \sim \Gamma^{t p}(i \mid e) \sim \Gamma^{t p}(e \mid i) \sim \Gamma^{t p}(e)$. Noting that the neoclassical heat flux $Q^{n c}(1) / T_{1}$ is given by $\Gamma^{t p}(1)$ up to 2 factor of order unity, this ordering contains some of the well-known important features of neoclassical theory, including the property of intrinsic ambipolarity. 


\section{Discussion}

In the foregoing sections, we have attempted to clarify the relationship between the ql and gBL transport theories, by focussing chiefly on the calculation of turbulently-induced rate $\dot{W}_{0}$ of interspecies energy exchange. The ql theory is a useful and much-used theory, but possesses certain serious limitations when applied to making statements about plasmas in their normal experimental state, supporting nearly-3teady-state turbulence. The gBL theory is a formally quite similar theory, preserving the ql virtue of analytic tractability, but which applies precisely for steady-state turbulence. It has been shown that $\dot{W}_{0}$ in the two theories are formally very similar, but that when evaluated in specific applications, can have very different magnitudes. for the same reasons as the particle and heat fluxes in neoclassical theory can differ greatly from the corresponding test-particle predictions. Using the CTEM for illustration, explicit expressions have been developed for $\dot{W}_{0}$ in the two theories, making these features concrete, as well as showing how the extra phase-space flux $\Gamma_{F}$ restores to the $g B L$ theory important properties lost in ql theory.

From the considerations presented in Sec. I, it is clear that ql theory applied to steady-state turbulence is deficient, dropping the flux $\Gamma_{F}$, which is of comparable size to the flux $\Gamma_{\mathrm{D}}$ kept. It is not clear that the 'pseudothermal' form for $\Gamma_{F}$ used by the gBL theory is a good representation of the form of $\Gamma_{F}$ which would result from a fully turbulent theory. However, the pseudothermal form used does preserve important properties which the fully turbulent $\Gamma_{F}$ must, and which ql theory sacrifices.

Assuming a thermal structure for the phase-space fluxes due to a tur- 
bulent spectrum occurs in the present work in two stages. First, in the original pseudothermal ansatz giving rise to the form for $\Gamma_{F}$ in Eqs. (18) and (19), and further, in the 'narrow-line-width' approximation adopted in Eq.( 33 ), to which much, though not all, of the subsequent development has been specialized. These pseudothermal assumptions have been used because they facilitate analytic progress, while preserving important features (such as conservation laws, the H-theorem, and the 'downhill' flow of energy) one knows a complete theory must possess. However, they also display further properties, such as Onsager symmetries, ${ }^{6}$ which intuition developed from past experience inclines one to expect should be true, but which may turn out not to be true for turbulent (as opposed to thermal) transport..$^{15,16}$

One particular place where the current pseudothermal form of the $g B L$ operator is likely to need improvement is in the dependence of the spectrum on the distribution $f(2)$ of driving species 2. In the current form of the $\mathrm{gBL}$ operator, the spectrum is computed, as for the standard BL operator, by superposing the uncorrelated contributions from individual test particles of species 2. This yields the undifferentiated $f_{0}$ in both terms in the gBL form (18), and leads to the relation $\psi(a, 2) \propto n_{2}$ in Eq.(31). Fully-turbulent, but more abstract, collision operators ${ }^{4,10}$ which have been developed display an analogous form, but with this discreteness-driven structure replaced by a turbulent correlation function which appears formally, and the calculation of which entails a formidable nonlinear calculation. Defining the field operator $\hat{\Phi}$ by $\delta \phi=\hat{\Phi} \delta f$ for any $\delta f$, one may formally express the spectrum appearing in the turbulent operator as $\left\langle|\delta \phi|^{2}\right\rangle=|\Delta|^{-2} \dot{\Phi} \tilde{C} \dot{\Phi}^{t}$ (superscript $t$ here denotes transpose), where $\tilde{C}(1,2) \equiv\left\langle\delta f^{(i)}(1) \delta f^{(i)}(2)\right\rangle$ is the correlation of the incoherent, 'unshielded' portion of $\delta f$ introduced in 
Sec. I. The discreteness-driven form for $\bar{C}$, from which the proportionality of $\left\langle|\delta \phi|^{2}\right\rangle$ or $\psi$ to $f_{0}$ arises, and whose adoption encapsulates the pseudothermal ansatz, is $\tilde{C}_{d}(1,2)=g f_{0}(1) \delta(1-2) g^{t}$. Using this form in the turbulent operator yields the standard BL operator for a uniform, unmagnetized plasma, ${ }^{4}$ and the gBL operator (18) for the more general range of configurations which it covers. Arguing heuristically that in the turbulent case, rather than the spectrum being driven predominantly by uncorrelated test particles, $\left\langle|\delta \phi|^{2}\right\rangle(2) \propto f_{0}(2)$, the spectrum is instead driven by the density $F_{0}(2)$ of 'macroparticles' of species 2 , suggests the replacement $f_{0} \rightarrow F_{0}$ in $\tilde{C}_{d}$ above. (Of course, the explicit calculation of $F_{0}$ would again entail the same difficult turbulence problem.) Adopting this new form yields an operator as in Eq.(18), but making the replacement $\left(\mathbf{l}_{1} \cdot \partial_{\mathbf{J}_{1}}-\mathbf{l}_{2} \cdot \partial_{\mathbf{J}_{2}}\right) f_{0}(1) f_{0}(2) \rightarrow \mathbf{l}_{1} \cdot \partial_{\mathbf{J}_{1}} f_{0}(1) F_{0}(2)-\mathbf{l}_{2} \cdot \partial_{\mathbf{J}_{2}} F_{0}(1) f_{0}(2)$ there. Following the same manipulations as used for the current gBL operator, ${ }^{5}$ it is easily shown that the new operator again conserves particles, angular momentum, and energy. However, it no longer possesses an H-theorem. Thus, it would appear that this particular prescription for improving on the current pseudothermal form of the gBL operator is insufficiently refined.

\section{Acknowledgments}

The author is grateful to W.M. Tang and J.A. Krommes for useful discussions relating to this work.

This work supported by U.S.Department of Energy Contract No.DEAC02-76-CHO3073. 


\section{References}

${ }^{1}$ A. Vedenov, E. Velikov, R.Z. Sagdeev, Nucl.Fusion 1, p.82 (1961)

${ }^{2}$ W.E. Drummond, D. Pines, Nucl.Fusion 1962 Suppl., Part 3, p.1049 (1962).

${ }^{3}$ A.N. Kaufman, J.Plasma Physics 8, Part 1, p:1 (1972).

${ }^{4}$ T.H.Dupree, Phys.Rev.Lett. 25, 789 (1970).

${ }^{5}$ H.E. Mynick,J.Plasma Phys. 39, Part 2, 303-317 (1988).

${ }^{6}$ H.E. Mynick an: R.E. Duvall, Phys. Fluids-B 1, 750 (1989).

${ }^{7}$ H..E. Mynick, Nucl.Fusion 30, 357 (1990).

${ }^{8}$ W.Horton, in Handbook of Plasma Physics, M.N. Rosenbluth and R.Z. Sagdeev (eds.), p.383 (Elsevier, New York, 1984).

${ }^{9}$ W.M. Tang, Princeton University Report PPPL-2728, (November, 1990).

${ }^{10}$ R.E. Duvall, H.E. Mynick, J.A. Krommes, Bubl.Am.Phys.Soc. 34,paper 6Q28 (1989).

${ }^{11}$ A. N. Kaufman, Phys. Fluids 15, 1063 (1972).

${ }^{12}$ D. A. Hitchcock, R. D. Hazeltine and S. M. Mahajan, Phys. Fluids 26, 2603 (1983).

${ }^{13}$ H. E. Mynick and J. A. Krommes, Phys. Fluids 23, 1229 (1980).

${ }^{14}$ R. D. Hazeltine, S. M. Mahajan and D. A. Hitchcock, Phys. Fluids 24, $1164(1981)$. 
${ }^{15}$ I.B. Bernstein, K. Molvig, Phys. Fluids 26, 1488 (1983).

${ }^{16} \mathrm{R}$. Balescu, Phys. Fluids B 3, 564 (1991). 
Dr. F. Pectoni, Univ. of Wollonoong. AUSTRALLA

Prot. M.H. Bromen, Univ. of Sychey, AUSTRALA

Pleama Pasearch Lob., Austrien Nat. Univ., AUSTRALLA

Prof. I.P. Jones, Finders Univ, AUSTRuLla

Prot, F. Cep, Inst for Theorotical Phyrics, AUSTRIA

Prof. M Hainder, meteut or Theoretwech Phycik, AUSTRLA

Prot. M. Goosens, Antronomiech Institure, BELGIUM

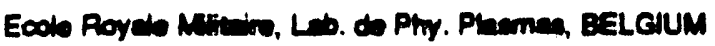

Commiscion European, DC XItFusion Proo., BELGIUM

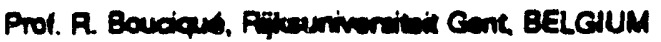

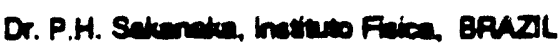

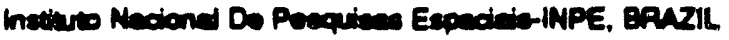

Dooumente Orios, Alomic Eneogy of Ceneda LAd., CANADA

Dr. MP. Bechynabi, MPB Technoberes, Inc., CANADA

Dr. H.R. Strangend, Univ. of Sademathemen, CAMAOA

Prof. J. Tedohmem, Univ, of Monered, CaNAOA

Prot. S.R. Sresivenen, Uriv. of Cedpry, CANAOA

Prot. T.W. Johneton, INASESnurgio, CAMADA

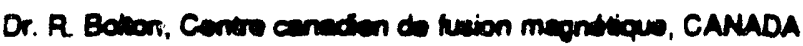

Dr. C.R. Jemes.. Univ. of Alusre, CANADA

D. P. Luktce, Kombendetho Univeresien, CZECHOSLOVAKIA

Tho Librerien, Cuhm Leboratory, ENGLAND

Librery. PB1, Ruthertord Appieton Leboracry, ENGLAND

Mrs. S.A. Hutchisison, JET Librery. ENGLAND

Dr. S.C. Shemen Univ. of South Pacific, FWI ISLANDS

P. Menonen, Uriv. of Halcinkj, FNLANO

Prot. M.N. Buasec, Ecols Pohychwique, FRANCE

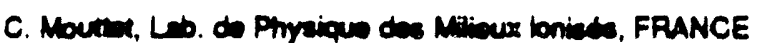

J. Redat CENCADARACHE - Bat 506, FPANCE

Prot. E. Economow, Univ. of Crew, GREECE

M. C. Pinni, Univ. of loannina, GAEECE

Dr. T. Mud, Academy Exiographic Ser., HONG KONG

Preprint Librey. Hengarien Acadmy of Sai., HUNGAPY

Dr. B. Descupten, Sana hat of Nucierer Physics, INDIA

Dr. P. Kan, Inst. bo Pleame Presearch, INOU

Dr. P. Powenes, lerey inst of Technology, ISPAEL

Libreien, Inimmationd Canter for Theo Phycics, ITALY

Mies C. Du Peb, Associerions EURATOMENEA, ITALY

D. G. Groseo, latiuts of Fivica ded Plasma, ITALY

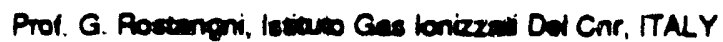

Dr. H. Yoneo, Tomina Res onver Center, JAPAN
Prot. I. Kamakemi, Hirochima Univ., LAPAN

Prof. K. Nichicemwe, Hiroahime Univ., JAPAN

Dirceor, Jepen Alowic Energy Resasarch Inst, JAPAN

Prot. S. Itoh, Kruatu Univ., LAPAN

Recaerch into. Cr., Natond Instit. for Fusion Scionce, JAPAN

Prot. S. Tenake, Kyow Univ., JAPAN

Librery, Kyoto Univ., JAPAN

Prot. N. Inoces, Univ. of Toloyo, LAPAN

Secretary, Pleame Section, Electrouchnical Lab., JAPAN

S. Mori, Techinied Advisor, MAEPA, JAPAN

Or. O. Mred Kunemos het of Technology. JAPAN

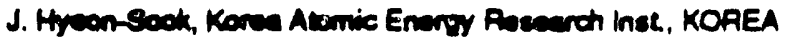

D.I. Chai, The Kores Adv. Inse of Sa. \& Tech., KOPEA

Prot. B.S. Liby, Univ. of Waitedo, NEW ZEALAND

Ines of Phyrica, Chineas Aad Sa PEOPLE'S REP. OF CHINA

Lurey, Inst of Piann Phyaica, PEOPLE'S REP. OF CHINA

Tringhua Univ. Lberery, PEOPLE'S REPUBUC OF CHIMA

$Z$ L, S.W. Ina Plyyica, PEOPLE'S REPUBUC OF CHINA

Prod. J.A.C. Gord, Inaituro Suparior Tecrico, POATUGAL

Dr. O. Potus, Al I CUzA Univ., ROMania

Dr. J. do Viviere, Fucion Sudies, AEC, S. AFAICA

Prof. M.A. Hellowo. Univ. of Nata, S. AFrica

Prot. D.E. Km, Poheng hat of Sa. 4 Tech., SO. KOREA

Prot. C.I.E.MA.T, Fuion Diviaion Library, SPAIN

Dr. L Simmo, Univ. of UMEA, SWEDEN

Lorery, Poye lnet of Technology, SWEDEN

Prot. H. Wrimimeon, Chaimers Univ. of Tech., SWEDEN

Centro Phys. Des Phemas, Eoob Pohyech, SWITZERLAND

Biblootsod, hat Voor Plasma-fycion, THE NETHERLANDS

Aase Prot. Dr. S. Catir, Midd Eant Tech. Univ., TURKEY

Dr. VA. Cultith,Sa. Res. Inte Enctrophys I Apparatus, USSA

Dr. D.D. Pyutov, Siberien Brench of Acadyny of Sai., USSR

Dr. GA. Einev, I.V. Kurchetor Inst, USSA

Libreien, Tho Ukr.SSR Academy of Scioncos, USSR

Or. LM. Kowizhnyth, Inse of Generd Phyzica, USSA

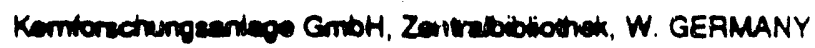

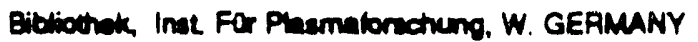

Prof. K. Schinder, Puhr-Univervit Bochum, W. GERMANY

Dr. F. Weoner. (ASOEX), Max-Plenck-thetitut, W. GEAMANY

Librarien, Max-Plenck-tnatiuc, W. GEAMANY

Prot. R.K. Jenev, Inot of Pirysices, YUGOSLAVIA 

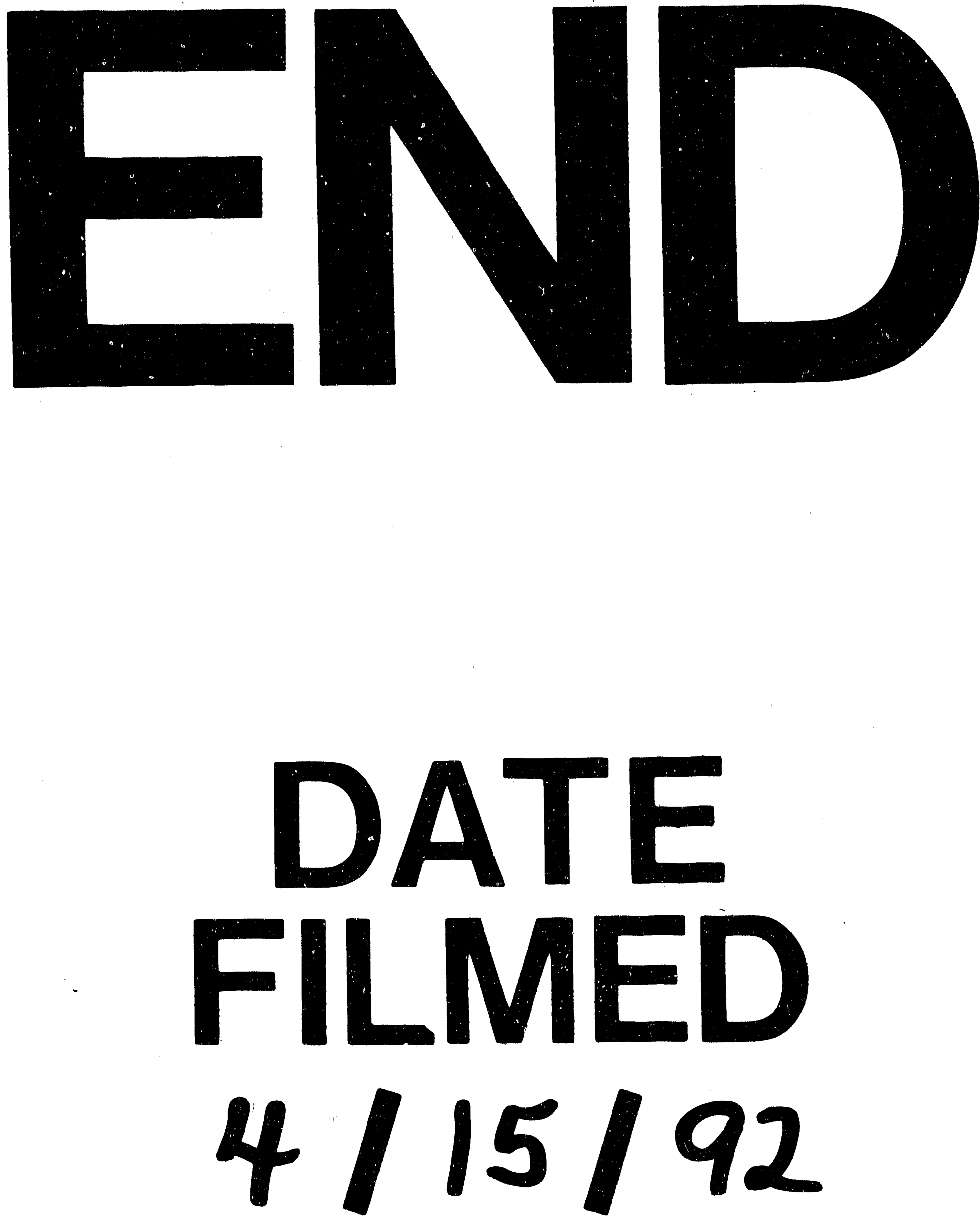
